\title{
On the Anisotropic Nature of MRI-driven Turbulence in Astrophysical Disks
}

Murphy, Gareth; Pessah, Martin E.

Published in:

Astrophysical Journal

DOI:

$10.1088 / 0004-637 X / 802 / 2 / 139$

Publication date:

2015

Document version

Publisher's PDF, also known as Version of record

Citation for published version (APA):

Murphy, G., \& Pessah, M. E. (2015). On the Anisotropic Nature of MRI-driven Turbulence in Astrophysical Disks.

Astrophysical Journal, 802(2), [139]. https://doi.org/10.1088/0004-637X/802/2/139 


\title{
ON THE ANISOTROPIC NATURE OF MRI-DRIVEN TURBULENCE IN ASTROPHYSICAL DISKS
}

\author{
Gareth C. Murphy and Martin E. Pessah \\ Niels Bohr International Academy, Niels Bohr Institute, Blegdamsvej 17, 2100 Copenhagen, Denmark; gmurphy@nbi.dk, mpessah@nbi.dk \\ Received 2014 October 22; accepted 2015 February 5; published 2015 April 2
}

\begin{abstract}
The magnetorotational instability (MRI) is thought to play an important role in enabling accretion in sufficiently ionized astrophysical disks. The rate at which MRI-driven turbulence transports angular momentum is intimately related to both the strength of the amplitudes of the fluctuations on various scales and the degree of anisotropy of the underlying turbulence. This has motivated several studies to characterize the distribution of turbulent power in spectral space. In this paper we investigate the anisotropic nature of MRI-driven turbulence using a pseudo-spectral code and introduce novel ways for providing a robust characterization of the underlying turbulence. We study the growth of the MRI and the subsequent transition to turbulence via parasitic instabilities, identifying their potential signature in the late linear stage. We show that the general flow properties vary in a quasi-periodic way on timescales comparable to $\sim 10$ inverse angular frequencies, motivating the temporal analysis of its anisotropy. We introduce a 3D tensor invariant analysis to quantify and classify the evolution of the anisotropy of the turbulent flow. This analysis shows a continuous high level of anisotropy, with brief sporadic transitions toward two- and three-component isotropic turbulent flow. This temporal-dependent anisotropy renders standard shell averaging especially when used simultaneously with long temporal averages, inadequate for characterizing MRI-driven turbulence. We propose an alternative way to extract spectral information from the turbulent magnetized flow, whose anisotropic character depends strongly on time. This consists of stacking 1D Fourier spectra along three orthogonal directions that exhibit maximum anisotropy in Fourier space. The resulting averaged spectra show that the power along each of the three independent directions differs by several orders of magnitude over most scales, except the largest ones. Our results suggest that a first-principles theory to describe fully developed MRI-driven turbulence will likely have to consider the anisotropic nature of the flow at a fundamental level.
\end{abstract}

Key words: accretion, accretion disks - black hole physics - instabilities - magnetohydrodynamics (MHD) turbulence

\section{INTRODUCTION}

The molecular viscosity operating in a laminar flow is too small by many orders of magnitude in order to account for the rate of angular momentum transfer inferred from observations of astrophysical disks (Frank et al. 2002). It has long been thought that turbulence offers a mechanism for efficient angular momentum transport in these disks (Shakura \& Sunyaev 1973; Lynden-Bell \& Pringle 1974). Regardless of its nature, turbulence in accretion disks must be anisotropic, since strictly isotropic turbulence is unable to lead to angular momentum transport.

In sufficiently ionized disks, the magnetorotational instability (MRI; Balbus \& Hawley 1991) offers a mechanism to drive magnetohydrodynamic (MHD) turbulence (Hawley et al. 1995; Brandenburg et al. 1996). In its most simple and powerful form, the MRI is a linear instability mediated by a weak vertical magnetic field that taps into the free energy of the differentially rotating flow, leading to exponential growth of the radial and azimuthal components of the velocity and magnetic fields. The correlated growth of these field components leads to transport of angular momentum mediated by Reynolds and Maxwell stresses, with the latter being the dominant, by a factor of a few in ideal MHD, in both the linear phase of the instability and the ensuing turbulence (Pessah et al. 2006).

There are two important properties of MRI-driven turbulence that influence the rate at which angular momentum is transported. One is clearly the strength of the fluctuations in the velocity and magnetic fields. Equally important, however, is the degree of anisotropy exhibited by the stress tensors associated with them, since completely isotropic tensors would lead to vanishing angular momentum transport. In the ideal MHD limit, the radial and azimuthal components of both the velocity and magnetic fields are exactly equal in the linear phase of the MRI and dominate over the vertical component. In the turbulent state, the azimuthal components dominate over their radial and vertical counterparts. This naturally creates a strong anisotropy in the turbulent flow. This suggests that the extent to which the flow is anisotropic evolves significantly from the linear phase of the MRI to the nonlinear, turbulent regime. Although there have been many studies of the linear phase of the MRI and its nonlinear evolution, only a relatively small fraction have explored in detail the mechanism responsible for its saturation, and none have focused explicitly on the evolution of the degree of anisotropy exhibited by the magnetized flow as it evolves from the linear regime of the instability to the ensuing turbulent state.

Motivated by the need to understand the processes at work, in this paper we analyze the saturation of the MRI and the ensuing anisotropic turbulence by means of targeted numerical experiments. This enables us to follow closely the evolution of the magnetized fluid through three main stages: the linear regime where the MRI grows unimpeded, the early nonlinear evolution leading to the turbulent state, and the fully developed turbulent regime. Aided by these simulations, we investigate how the amplitude of the fluctuations and the degree of anisotropy evolve throughout by employing statistical tools that have not been applied to MRI-driven turbulence before.

In order to extract statistical information about the properties of MRI-driven turbulence, most previous works have focused on using spherical shell averages in spectral space, which is 
suitable for isotropic turbulence. However, the underlying spectral distributions in MRI-driven turbulence are far from isotropic. This suggests that the use of spherical shell averages may not be optimal for analyzing and representing spectral energy distributions in MRI-driven turbulence. As a viable alternative, we employ a joint approach that consists of using invariant maps for measuring anisotropy in real space and dissecting the three-dimensional (3D) Fourier spectrum along the most relevant planes, as revealed by the anisotropic nature of the flow. We envisage that the new insights offered by this approach will be valuable for guiding a more fundamental understanding of the anisotropic nature of MHD turbulence in astrophysical disks.

The paper is organized as follows. In Section 2 we provide an overview of previous works devoted to analyzing the mechanisms that lead to the saturation of the MRI. We motivate the use of targeted numerical experiments to shed light on the processes involved when the agent responsible for saturation is related to parasitic instabilities. In Section 3 we examine the Kelvin-Helmholtz $(\mathrm{KH})$ instability of a sinusoidal velocity field in order to prepare the ground to study the development and saturation of the MRI due to secondary instabilities in Section 4. In Section 5 we introduce the concepts involved in invariant analysis introduced by Lumley \& Newman (1977) and use them to study the evolution of anisotropy of the MRI-driven turbulent flow. As a complementary approach, we analyze the evolution of the 3D spectral distribution characterizing the energy density and the Maxwell stress in Fourier space, from the early phases of the nonlinear stage of the MRI to the fully developed turbulent state. By carefully stacking power spectra along the principal directions characterizing the turbulent state, we define a more meaningful way of describing its anisotropic nature. We summarize our results and discuss their implications in Section 6.

\section{PREVIOUS WORKS ON THE SATURATION OF THE MRI}

Several different approaches have been taken in the literature to investigate the mechanisms responsible for the saturation of the MRI. One group of works has focused on understanding the role of parasitic instabilities that feed off the primary MRI mode in the incompressible MHD regime using analytical (Goodman \& Xu 1994; Pessah \& Goodman 2009; Pessah 2010) and numerical (Latter et al. 2009; Longaretti \& Lesur 2010) means, while others (Latter et al. 2009; Obergaulinger et al. 2009) have relaxed the assumption of an incompressible flow and used numerical simulations to explore how the instability saturates. All of these studies have been carried out in the framework of the shearing sheet, where, by definition, the background flow, and in particular the shear profile feeding the MRI, remains unaltered. This is probably a reasonable assumption in astrophysical settings where the ultimate source of energy responsible for differential rotation (i.e., the central potential) remains unchanged.

A second approach has focused on the saturation of the MRI due to flattening of the background shear profile. This route to suppress the energy source for the instability is present in settings where the shear profile is not imposed throughout the flow, as is the case when rotating rigid walls are considered. A series of papers by Knobloch \& Julien (2005), Julien \& Knobloch (2006, 2010), Jamroz et al. (2008a, 2008b), and Tatsuno \& Dorland (2008) find that in laboratory settings, the
MRI saturated state is not turbulent but consists of laminar axisymmetric layers threaded by magnetic fields with alternating polarity (see, e.g., Figure 4 in Jamroz et al. 2008b). The initial background shear profile is modified and approaches solid-body rotation, leading to a laminar saturated state that settles on a resistive timescale. Tatsuno \& Dorland (2008) carried out two-dimensional (2D) simulations with rigid boundaries, finding that the initial Keplerian background profile becomes flattened (see, e.g., their Figure 5). In this study, parasite modes related to $\mathrm{KH}$ instabilities are not detected, although they do detect tearing modes that lead to the formation of magnetic islands between laminar magnetic layers.

Other approaches have focused on saturation via diversion of energy into magnetosonic waves, or generation of large-scale magnetic fields via a dynamo process. Liverts et al. (2012) suggested that the MRI saturates by driving nonresonant magnetosonic waves in a bursty oscillatory manner. Umurhan et al. (2007) perform a weakly nonlinear analysis of the MRI and find that it saturates with only a modest reduction in background shear, with an amplitude proportional to the square root of the magnetic Prandtl number, provided that this is much smaller than unity. Vishniac (2009) suggested that, in the absence of net magnetic flux, the MRI dynamo saturation level is controlled by the imposition of a maximum wavenumber on MRI modes due to the combined effects of magnetic tension, buoyancy, and/or resistivity or viscosity. Alternatively, Ebrahimi et al. (2009) propose that the MRI saturates by generating large-scale fields through an $\alpha$-effect, although they point out that, since the total toroidal and axial flux does not change significantly with time, this cannot be referred to as a largescale dynamo effect.

In this work, we focus on parasite modes as a potential cause of saturation. The notion that the exponentially growing MRI modes are themselves, in turn, subject to secondary, parasitic instabilities was originally suggested by Goodman \& Xu (1994) and has been considered in more detail by a number of works more recently (Bodo et al. 2008; Latter et al. 2009; Obergaulinger et al. 2009; Pessah \& Goodman 2009; Pessah 2010). The physical arguments that support the development of secondary instabilities are quite robust. Provided that the initial magnetic field is very weak, the MRI modes are exact solutions of the equations of motion and thus can reach large amplitudes. These modes lead to exponentially growing velocity and magnetic fields that vary sinusoidally in the vertical direction (perpendicular to the disk midplane). When the amplitude of these perturbations is large enough, they become themselves subject to secondary instabilities. Broadly speaking, these secondary parasitic modes belong to two classes related to the $\mathrm{KH}$ instability and the tearing mode (TM) instability. As discussed in Pessah (2010), the KH parasitic mode dominates in regimes near ideal MHD, appropriate for highly ionized accretion disks, whereas the TM parasite dominates in highly resistive regimes, such as laboratory experiments and protoplanetary disks. Since these secondary instabilities extract energy from the MRI, they are expected to limit its growth and to potentially cause its saturation.

Because of the complexity inherently involved in carrying out secondary instability analyses, previous semi-analytical works have made a number of approximations. In particular, the exact (primary) MRI modes are considered as a timeindependent background off of which the (secondary) parasitic 
modes feed. The effects of the weak vertical background field, the Coriolis force, and the background shear flow are all usually neglected in the equations of motion governing the dynamics of the secondary instabilities (Goodman \& Xu 1994; Pessah \& Goodman 2009). Relaxing these approximations in analytical studies is a challenging task; therefore, we have designed tailored numerical experiments that allow us to follow in detail the evolution of the flow from the early onset of the instability until the fully development turbulent regime ensues. The numerical experiment that we consider below isolates the fastest-growing, axisymmetric MRI mode and follows its evolution after seeding the perturbations that excite a nonaxisymmetric $\mathrm{KH}$ parasitic mode of the type that are expected to exhibit the fastest growth according to semianalytical studies (Pessah 2010). This controlled experiment allows to us address the evolution of the degree of anisotropy in the flow from the early linear phase of the MRI until the fully developed turbulent state.

\section{EXACT MRI MODES AND RELATED KH PARASITES}

\subsection{Equations and Fastest-growing Modes}

The equations governing the local dynamics of a magnetized disk in the shearing box framework are given by (Hawley et al. 1995; Brandenburg et al. 1996)

$$
\begin{aligned}
& \frac{\partial \boldsymbol{v}}{\partial t}+\boldsymbol{v} \cdot \nabla \boldsymbol{v}=-2 \Omega \times \boldsymbol{v}+2 q \Omega^{2} x \\
&-\frac{\boldsymbol{\nabla p}}{\rho}+\frac{\boldsymbol{J} \times \boldsymbol{B}}{\rho}+\nu \nabla^{2} v, \\
& \frac{\partial \boldsymbol{B}}{\partial t}=\nabla \times(\boldsymbol{v} \times \boldsymbol{B})+\eta \nabla^{2} \boldsymbol{B} .
\end{aligned}
$$

Here $\rho$ is the mass density, $v$ is the fluid velocity in the rotating frame, with angular frequency $\Omega=\Omega \hat{z}, p$ is the pressure determined through an equation of state, and $\boldsymbol{B}$ is the magnetic field, with $\nabla \cdot \boldsymbol{B}=0$. The current density is $\boldsymbol{J} \equiv \nabla \times \boldsymbol{B} / \mu_{0}$, with $\mu_{0}$ a constant dependent on the unit system adopted. The first and second terms on the right-hand side of Equation (1) account for the Coriolis acceleration and the first-order expansion of the tidal potential, respectively. The shear parameter is defined as

$$
q=-\frac{d \ln \Omega}{d \ln r}
$$

so that $q=3 / 2$ for a Keplerian disk. The coefficients $\nu$ and $\eta$ stand for the viscosity and resistivity, assumed to be constant.

Let us consider an incompressible flow, which is a good approximation provided that the magnetic field involved is very weak, i.e., the magnetic energy density is small compared to its thermal counterpart. The set of Equations (1)-(2) admits exact, MRI-unstable solutions of the form

$$
\begin{gathered}
\boldsymbol{v}=-q \Omega x \check{\boldsymbol{y}}+\boldsymbol{V}_{0} \sin (K z) e^{\Gamma t}, \\
\boldsymbol{B}=\bar{B}_{z} \check{z}+\boldsymbol{B}_{0} \cos (K z) e^{\Gamma t} .
\end{gathered}
$$

The ratio of the amplitudes, i.e., $B_{0} / V_{0}$, sets the initial amplitude of the MRI mode. In ideal MHD, the wavelength of the fastest mode is $\lambda_{\max }=2 \pi / K_{\max }=\sqrt{15 / 16} \bar{v}_{A, z} / \Omega$, where $\bar{v}_{A, z}=\bar{B}_{z} / \sqrt{4 \pi \rho}$ is the Alfvén speed associated with the background magnetic field, $\bar{B}_{z}$. This mode grows at the rate $\Gamma_{\max }=3 \Omega / 4$, with $V_{A, 0} / V_{0}=\sqrt{5 / 3}$, where $V_{A, 0}=B_{0} / \sqrt{4 \pi \rho}$ is the Alfvén speed associated with the MRI-driven perturbation (Pessah et al. 2006).

These exact primary MRI modes are themselves, in turn, subject to secondary, parasitic instabilities. The fastest-growing parasitic modes, which are associated with $\mathrm{KH}$ modes, have the same vertical periodicity as the primary MRI mode itself (Goodman \& Xu 1994; Pessah \& Goodman 2009). As a prelude to analyzing in detail the nonlinear evolution of the MRI modes as they are affected by secondary instabilities, we perform a series of simpler numerical experiments to study the dynamics of the $\mathrm{KH}$ instabilities resulting from a periodic velocity profile with constant amplitude.

\subsection{KH Modes on a Sinusoidal Velocity Field}

We begin by examining the development of $\mathrm{KH}$ modes that results from a background flow with $\delta v_{x}=V_{0} \sin \left(2 \pi z / L_{z}\right)$. This velocity profile is akin to the velocity profile induced by an MRI mode, except for the fact that its amplitude is time independent. Incidentally, note that a time-independent MRI amplitude is usually invoked when calculating secondary instabilities affecting an MRI mode (Goodman \& Xu 1994; Latter et al. 2009; Pessah 2010). The argument behind this approximation is that, provided that the amplitude of the MRI is large enough, the growth rate of the secondary instability should be much larger than the MRI growth rate. This preliminary exercise is useful for two reasons. It provides an independent check for the semi-analytical results predicting the parasitic growth rates (Goodman \& Xu 1994) and mode structure (Pessah 2010). It allows us to get acquainted with the type of mode structure that emerges as a natural consequence of the action of parasitic modes in 3D shearing-box simulations of the MRI discussed below.

We solve the set of equations describing a hydrodynamic flow starting from the initial conditions

$$
\boldsymbol{v}(x, z)=\left(\begin{array}{c}
V_{0} \sin (K z) \\
0 \\
\epsilon V_{0} \sin \left(k_{h} x_{h}\right)
\end{array}\right),
$$

where $\epsilon=10^{-2}$. The stability of this flow can be analyzed semi-analytically, as described in Goodman \& Xu (1994) and Pessah (2010). The flow is unstable with a dimensionless growth rate $s / K V_{0}$ that depends only on $k_{h} / K$, i.e., the ratio of the wavenumbers associated with the periodic background shear flow and the excited perturbation. This growth rate attains a maximum at $k_{h} / K \simeq 0.6$ and vanishes beyond $k_{h} / K=1$. This is illustrated in Figure 1, where we compare the results obtained with linear theory and numerical codes. In order to gauge the importance of compressibility in the growth of the KH instabilities, we performed two separate sets of simulations in compressible and incompressible gas dynamics using the publicly available codes PLUTO (Mignone et al. 2012; where the equation of state is isothermal) and SNOOPY (Lesur \& Longaretti 2009). As expected, the results of the spectral code SNOOPY are closer to the analytical results than the finitevolume code PLUTO; the difference is accounted for by compressibility.

The early evolution of this sinusoidal, 2D flow leads to the development of sheets of cellular vortices of alternating polarities in both radius and height, as shown in Figure 2. The agreement between the analytical and numerical results in the linear regime is also excellent in terms of the structures 


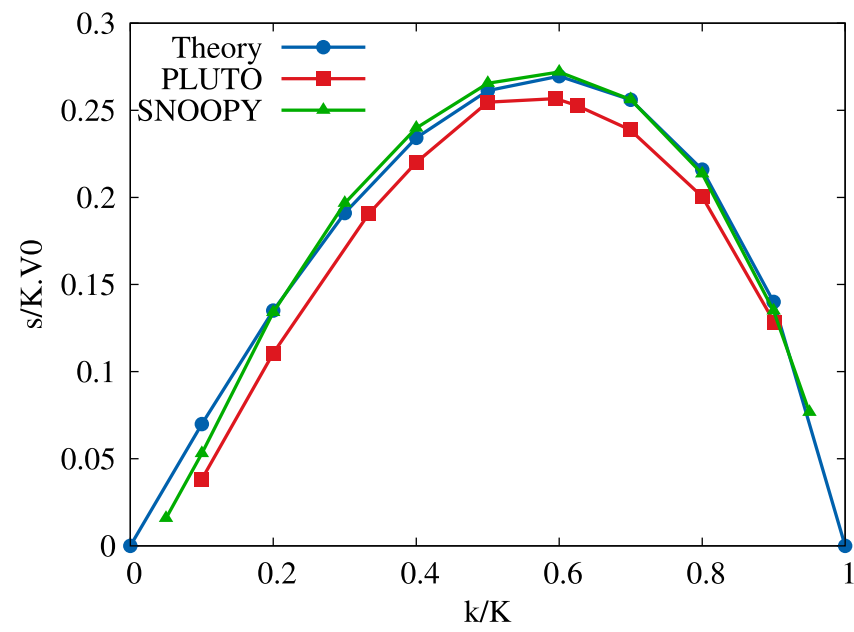

Figure 1. Comparison of the dimensionless growth rates for $\mathrm{KH}$ instabilities excited by a sinusoidal velocity profile as a function of $k_{h} / K$, i.e., the ratio of the wavenumbers associated with the periodic background shear flow and the perturbations. The pseudo-spectral code SNOOPY agrees most closely with the theoretical results derived for an incompressible flow. The results obtained with the PLUTO code show that the effect of compressibility is to slightly reduce the growth rates expected from analytical theory.

formed. The extremum of the vorticity distribution is due to a combination of circulation and shear vorticity, so the peak of the color map does not coincide with the point around which the fluid seems to be circulating. Figure 3 shows the evolution of the instability into the nonlinear regime, where the vortices interact, grow, and merge.

The saturated state, depicted in the rightmost panel of Figure 3, consists of episodic fluctuations in the vertical component of the velocity, which leads to a decrease of $v_{z}^{2}$ to about $50 \%$ of its initial value. The radial component of the velocity also shows episodic fluctuations, which leads $v_{x}^{2}$ to increase to $80 \%$ of the initial kinetic energy (Figure 3). At later times, shock waves form, fine sheared structures appear, and kinetic energy is dissipated into thermal energy. Note that while the initial shear flow is aligned in the $x$-direction, the $\mathrm{KH}$ instability increases the flow speed in the transverse, $z-$ direction, until it reaches the same order of magnitude, so that initially anisotropic flow becomes partially isotropized.

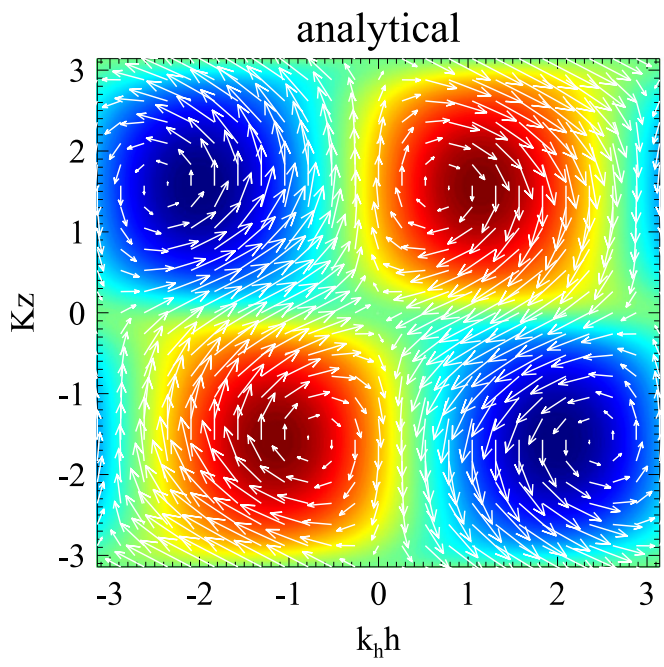

\section{DEVELOPMENT AND SATURATION OF THE MRI}

\subsection{Beyond semi-analytical Approximations}

In the standard picture, parasitic instabilities are thought to feed off the exponentially growing background provided by the primary MRI mode, which corresponds to an exact solution of the MHD equations provided that compressibility is unimportant. In ideal MHD, the growth rate of these secondary instabilities is expected to be proportional to the amplitude of the MRI mode, and thus the growth of the parasites is superexponential. Therefore, the energy obtained by the secondary instabilities can become comparable to the energy in the MRI mode from which it feeds very quickly. Because the energy going into secondary instabilities is envisioned to be directly supplied by the primary mode, the fast growth of the secondary could potentially prevent the MRI from continuing growing, halting its exponential growth and leading thus to its saturation.

This picture assumes that the amplitude of the MRI is large enough so that several approximations can be invoked. Perhaps more importantly, it also assumes that the dynamics of the secondary perturbations is insensitive to the shearing background and that the MRI grows uninhibited. The first approximation implies that the parasitic perturbations can be described by a time-independent wavevector $\boldsymbol{k}_{h}=\left(k_{x}, k_{y}\right)$, which characterizes the wavelength of the perturbations in horizontal planes, i.e., perpendicular to z-direction. However, because of advection by the background shear flow, the wavevectors of nonaxisymmetric perturbations will evolve in time according to $k_{x}(t)=k_{x}-q \Omega t k_{y}$. The second approximation prevents any dynamic coupling between the primary mode and the secondary parasites. This approximation becomes increasingly worse as the amplitude of the parasite grows larger. In order to understand the role of secondary instabilities and how they mediate the transfer of power from axisymmetric MRI modes into nonaxisymmetric structures, we set up a tailored numerical experiment that is not limited by the assumptions usually invoked in the semi-analytical studies described above. For the sake of simplicity, we focus on following the dynamical evolution of the fastest-growing MRI mode.

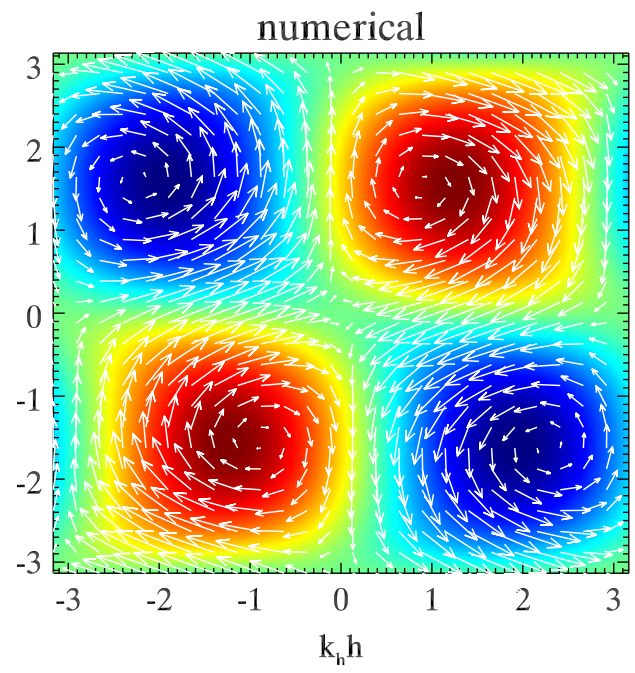

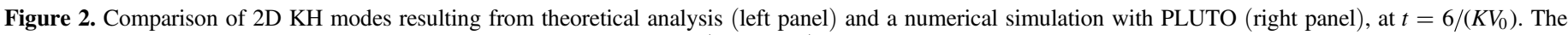

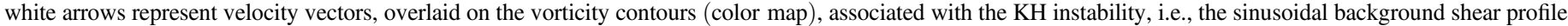
has been subtracted. The plots show almost identical flow features, with similar peaks in the vorticity and offset centers of the circulating velocity vectors. 

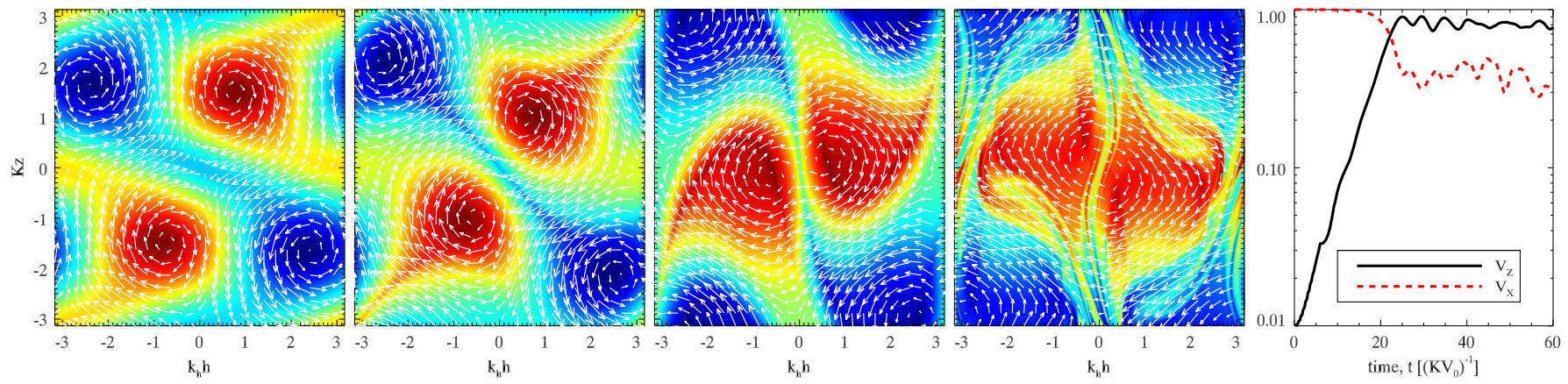

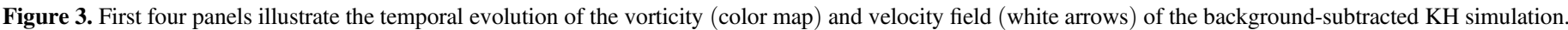

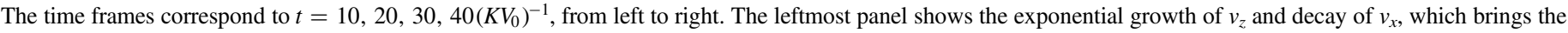
flow closer to an isotropic state.

\subsection{Tailored Numerical Setup}

The incompressible simulations are carried out using the SNOOPY spectral code (Lesur \& Longaretti 2009), which solves the MHD equations in the constant-density regime and incorporates a finite physical resistivity and viscosity. The resistivity is such that $\Lambda_{\eta} \equiv \bar{v}_{A z}^{2} / \eta \Omega=10^{4}$, and the magnetic Prandtl number is unity. For practical purposes, this is rather close to ideal MHD and corresponds to the regime where the secondary $\mathrm{KH}$ mode is expected to dominate.

We set up the numerical experiment as follows. In order to maximize the numerical resolution, we set the vertical extent of the simulation domain to be equal to the most unstable MRI wavelength, so that $L_{z}=2 \pi K_{\max }=\sqrt{15 / 16} \bar{v}_{A, z} / \Omega$. This motivates choosing $L_{z}$ and $\Omega^{-1}$ as the units of length and time. All the results in the paper correspond to a simulation with a resolution of $256 \times 256 \times 128$ cells, which was carried out for $260 \Omega^{-1}$. The only exception are the results where we used the lower resolution of $128 \times 128 \times 64$ cells in order to run the simulation for $400 \Omega^{-1}$.

We initialize the simulation by exciting the fastest-growing MRI mode at $t=0$, i.e.,

$$
\begin{gathered}
\boldsymbol{v}(x, y, z, t=0)=\delta v\left(\begin{array}{c}
\sin (K z) \\
\sin (K z) \\
0
\end{array}\right), \\
\boldsymbol{B}(x, y, z, t=0)=\delta B\left(\begin{array}{c}
\cos (K z) \\
-\cos (K z) \\
0
\end{array}\right),
\end{gathered}
$$

where $\delta v=10^{-3} L_{z} \Omega$ and $\delta B / \sqrt{4 \pi \rho}=\sqrt{5 / 3} \delta v$. As expected, this initial perturbation grows exponentially at a rate $\Gamma_{\max }=3 / 4 \Omega$ (Figure 4 ) with the mode structure predicted by linear theory. In incompressible dynamics this exponential growth can continue for several orbits unless the fluid is perturbed.

In order to break down the exponential growth of the MRI, we seed a perturbation with a horizontal wavevector given by $\boldsymbol{k}_{h}=(1,1,0) .{ }^{1}$ Note that this wavevector is chosen in the direction where the shear induced by the MRI is fastest, i.e., at $45^{\circ}$ with respect to the radial direction. Because this mode is nonaxisymmetric, its excitation requires some care for the

\footnotetext{
1 Note that the horizontal wavenumber $k_{h} / K \simeq 0.7$ is chosen to ensure compatibility of the periodic boundaries in horizontal planes, whereas the fastest-growing parasite is $k_{h} / K \simeq 0.6$, according to semi-analytical studies.
}

following reason. If a nonaxisymmetric perturbation is excited at time $t=0$, since the radial wavenumber has a linear dependence on time, it reaches the Nyquist wavenumber in $t_{\text {alias }}=\log _{2}\left(N_{x, g}\right) \Omega^{-1}$ (where $N_{x, g}$ is the number of grid cells in the $x$-direction), where it is aliased to $k_{x}=1$ before it can grow significantly (Lyra \& Klahr 2011). To circumvent this, we excite the perturbation

$$
\delta \boldsymbol{v}(x, y, z, t=6)=\epsilon \delta v \sin \left(\boldsymbol{k}_{h} \cdot \boldsymbol{x}\right) \check{z},
$$

with $\epsilon=10^{-4}$ at time $t=6 \Omega^{-1}$. This allows the perturbation to seed the parasitic mode, avoiding the effects of aliasing due to finite grid resolution. This secondary instability grows superexponentially (Figure 4), exhibiting the characteristics expected of a $\mathrm{KH}$ instability feeding off the velocity shear induced by the primary MRI mode (see Figure 5 and Pessah 2010). The subsequent evolution of this 3D, nonaxisymmetric perturbation, in the plane parallel to $\boldsymbol{k}_{h}$, i.e., where the MRI-induced velocity shear is largest, is shown in Figure 6. Note that the rightmost panel there shows a close-up of Figure 4 at the time where the amplitude of the secondary instability approaches that of the MRI.

In order to analyze the dynamics in detail, it is useful to divide the evolution of the flow into three stages: (i) an exponential growth phase driven by the MRI in which the amplitude of the fast-growing parasite is too small to modify the primary mode; (ii) a transitionstage in which the energy of the parasite is comparable to the energy of the primary MRI mode, which starts to depart significantly from the initial exact solution; and (iii) a turbulent state in which all flow variables fluctuate in time. In what follows, we describe briefly each of these stages.

\subsection{MRI Exponential Growth in the Linear Regime}

The initial conditions in Equations (7) and (8) lead to exponential growth of the exact solution that grows for several $\Omega^{-1}$ timescales. The amplitude of this perturbation grows by more than two orders of magnitude, retaining the initial MRI mode structure in which the sinusoidal velocity field, with $\delta v_{x}=\delta v_{y}$, is orthogonal to the co-sinusoidal magnetic field perturbations, with $\delta B_{x}=\delta B_{y}$. This is illustrated in the leftmost panel of Figure 7, which shows the magnitude of the radial and azimuthal components of the velocity and magnetic field (Pessah \& Chan 2008) as a scatter plot (see, e.g., Bodo et al. 2008). 


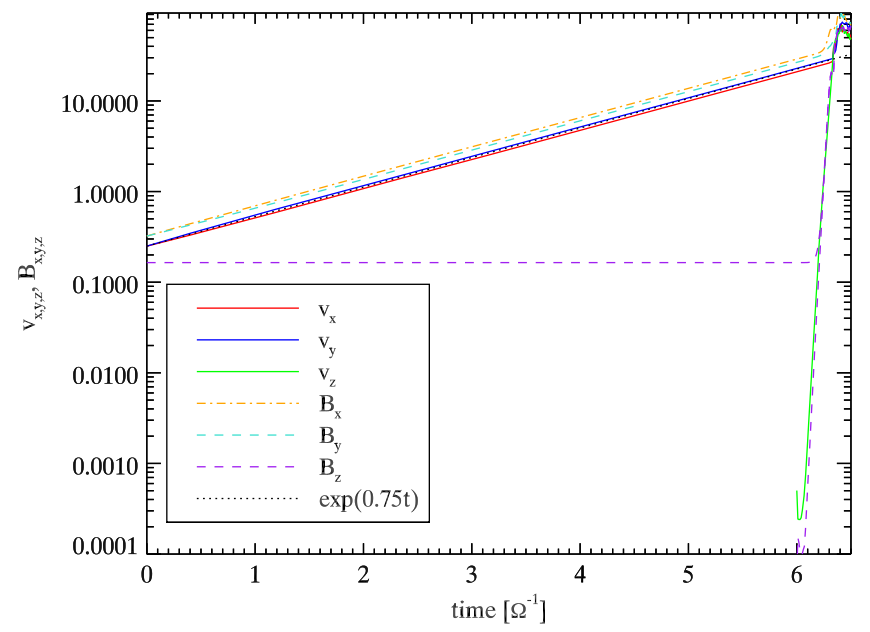

Figure 4. Time evolution of integrated quantities $B_{x, y, z}$ and $v_{x, y, z}$ plotted with a fit to the MRI growth rate of $0.75 \Omega$. The field components $v_{x, y}$ and $B_{x, y}$ grow exponentially, while $B_{z}$ remains constant until $t=6 \Omega^{-1}$, when $v_{z}$ is perturbed and then grows superexponentially.

In the incompressible regime under consideration, the exact MRI mode does not lead to velocity or magnetic field perturbations in the vertical direction. Therefore, keeping track of $\delta v_{z}$ and $\delta B_{z}$ allows us to quantify how the evolution of the flow departs from the exact MRI mode excited at $t=0$. Figure 4 illustrates that the vertical component of the magnetic field, which gives rise to the MRI, remains constant until the secondary perturbation (9) is excited at $t=6 \Omega^{-1}$. The initial perturbation seeding $\delta v_{z}$ grows superexponentially together with $\delta B_{z}$, affecting all other field components, which start to depart significantly from the MRI behavior predicted by linear theory soon after. This is also evident from the middle and rightmost panels in Figure 7.

The shear resulting from the exponentially growing sinusoidal velocity field that results from the MRI is maximum along the direction $x=y$, which is the direction in which we have chosen $\boldsymbol{k}_{h}$. This plays an important role in the 3D, nonaxisymmetric nature of the parasitic modes. The evolution of the parasitic mode is illustrated in Figure 6. The first four panels, from left to right, show the projected component of the vorticity into the $\left(x_{h}, z\right)$ plane, with $x_{h}=\boldsymbol{x} \cdot \boldsymbol{k}_{h}$. The initial vorticity perturbation, which is of the form $\nabla \times \boldsymbol{v}_{h} \propto \cos \left(\boldsymbol{k}_{h} \cdot \boldsymbol{x}\right) \hat{\boldsymbol{k}}_{p}$, with $\hat{\boldsymbol{k}}_{p}=\hat{\boldsymbol{k}}_{z} \times \hat{\boldsymbol{k}}_{h}$, gives rise to a sheet of cellular vortices, as predicted in Pessah (2010). The first and second panels, from left to right, show that the cellular vortices begin to interact in the nonlinear stage and vorticity concentrates near the boundary of the vortex sheets, at $K z=0, \pm 0.5$. The sheets of vortices can be seen in the 3D slices of vorticity and velocity field lines projected into the $\left(x_{h}, z\right)$ plane (see Figure 5), where the familiar parasite structure exhibits extrema visible at $z=-0.25,0.25$.

\subsection{Takeover by Parasitic Instabilities}

The vorticity plots in the third and fourth panels of Figure 6 illustrate how the interface undulations roll over into the classical cat's eye associated with $\mathrm{KH}$ vortices, which appears between the two shearing layers, similar to those seen in Frank et al. (1996), Jones et al. (1997), and Malagoli et al. (1996). At this time $\left(6.3-6.4 \Omega^{-1}\right)$ the peak value of the magnetic field $B_{z}$ exceeds the peak value of the vertical velocity component, $v_{z}$.

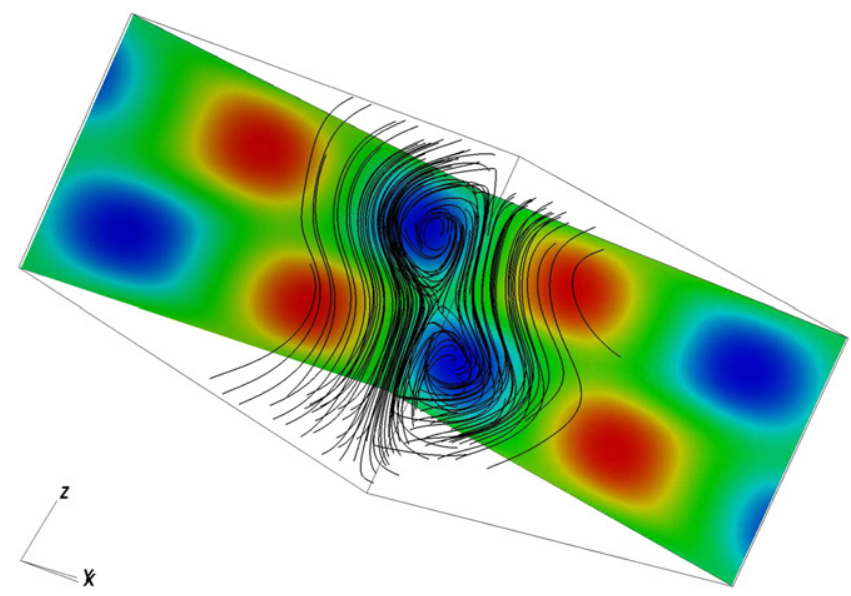

Figure 5. Vorticity (color map) with exponentially growing MRI background subtracted and projected into the $x_{p}$ direction at $t=6.05 \Omega^{-1}$. The slices are in the $x, z$ and $y, z$ planes. The black solid lines show the magnetic field.

The radial component of the magnetic field has also started to depart from its linear growth and exceeds the MRI growth rate, as can be seen from Figure 4. As a result of the superexponential growth of the KH instability, the flow becomes completely disrupted and transitions rapidly into a turbulent state.

It is instructive to compare the evolution of the secondary $\mathrm{KH}$ instabilities that feed off the exponentially growing sinusoidal shear provided by the MRI and the constant, imposed sinusoidal shear addressed in Section 3.2. Because the growth of the MRI mode induces magnetic field perturbations that are orthogonal to the velocity fluctuations, the nature of the motions in the plane $\left(x_{h}, z\right)$ is essentially hydrodynamic. Therefore, it is not surprising that the perturbations (9) lead to the excitation of $\mathrm{KH}$ instabilities similar to the ones described in Section 3.2. However, because the amplitude of the velocity shear off of which the secondary instability feeds is growing exponentially in time, their late temporal evolution exhibits significant differences with respect to the $\mathrm{KH}$ instabilities triggered by the constant-amplitude sinusoidal profile (compare Figures 3 and 6).

In the linear stage of the MRI, the flow is strongly anisotropic, as the vertical components of the magnetic field and velocity are greatly exceeded by their radial and axial counterparts. The departure from the linear state can be seen in the scatter plots of $B_{x, y}$ and $v_{x, y}$ (see Figure 7); the second panel shows the departure from the linear mode at $t=6.5 \Omega^{-1}$, shortly after the perturbation was excited. The magnetic field components $B_{x}$ and $B_{y}$ no longer increase exponentially, but instead a parasite mode starts to take over. The $B_{x}, B_{y}$ structures, which grow at right angles to the initial MRI flow, at $\left(B_{x}, B_{y}\right)=(-2,1)$ and $(2,-1)$ in the second panel, then correspond to the growth of parasitic modes. At saturation time, all three components of the velocity and magnetic fields are approaching the same magnitude. In essence, the growth of parasite modes has a strong effect on the anisotropy of the flow, reducing it and attempting to return to isotropy, as can also be seen in Figure 7.

The MRI mode starts to saturate as energy is drained from the radial and azimuthal components of velocity and magnetic fields and fed into the vertical components of velocity and magnetic fields. Thus, a reduction in the strong anisotropy, 
from $v_{x} \sim v_{y} \gg v_{z}$ to $v_{x} \sim v_{y} \sim v_{z}$, appears to accompany the parasite modal growth.

\subsection{Quasi-periodic Turbulent State}

After the parasite mode has grown to its maximum value at $t=6.6 \Omega^{-1}$, and several $\Omega^{-1}$ have elapsed, a state of fully developed, anisotropic turbulence is reached. The analysis of the MRI-driven turbulent state has been the subject of a large body of literature. Here we point out that the turbulent regime observed in our numerical experiment is characterized by sequences of peaks in the $v_{x}$ component followed by peaks in $v_{z}$. These quasi-periodic oscillations are evident in Figure 8. The radial component $v_{x}$ always increases more slowly, followed by a rapid increase in the vertical component $v_{z}$. As both components attain their respective local maxima, they decrease in tandem and at the same rate. Interestingly, this echoes the behavior seen in the linear stage, where the growth in $v_{x}$ and $v_{y}$ (driven by the MRI mode) triggers the growth in $v_{z}$ (resulting from the excitation of parasite modes), which then prevents the amplitude of the MRI from growing further.

In order to quantify this periodicity, we analyze the time series provided by $v_{x}(t)$. We consider a total of $350 \Omega^{-1}$, discarding the initial $50 \Omega^{-1}$ in which the simulation is growing exponentially and then transitioning toward fully turbulent flow. We use Bartlett's method of estimating power spectra via averaging periodograms (Bartlett 1948) in order to reduce their variance. This is achieved by splitting the time series for $v_{x}(50<t<300)$ into four independent samples, calculating the Fourier amplitudes of these time series, and averaging their square for each frequency (see Figure 8). The bulk of the power is concentrated between 9 and $13 \Omega^{-1}$. A spike is also seen at two-thirds of a period, which corresponds to one shearing time.

In the saturated state, the flow is characterized by highly anisotropic turbulence, which exhibits quasi-episodic bursts. Bodo et al. (2008) attributed the presence (absence) of these bursts to the absence (presence) of parasite modes. In the linear stage, it has been possible to directly subtract the background MRI exact solution, in order to identify signatures and compare with those predicted in semianalytical calculations (Pessah 2010). However, in the turbulent state, the same approach becomes more difficult. Exact solutions are not easy to identify or subtract in a consistent way. Therefore, we pursue an alternative approach, in order to engage directly with the fluctuations in the Reynolds and Maxwell stresses that are associated with angular momentum transport in the turbulent state.
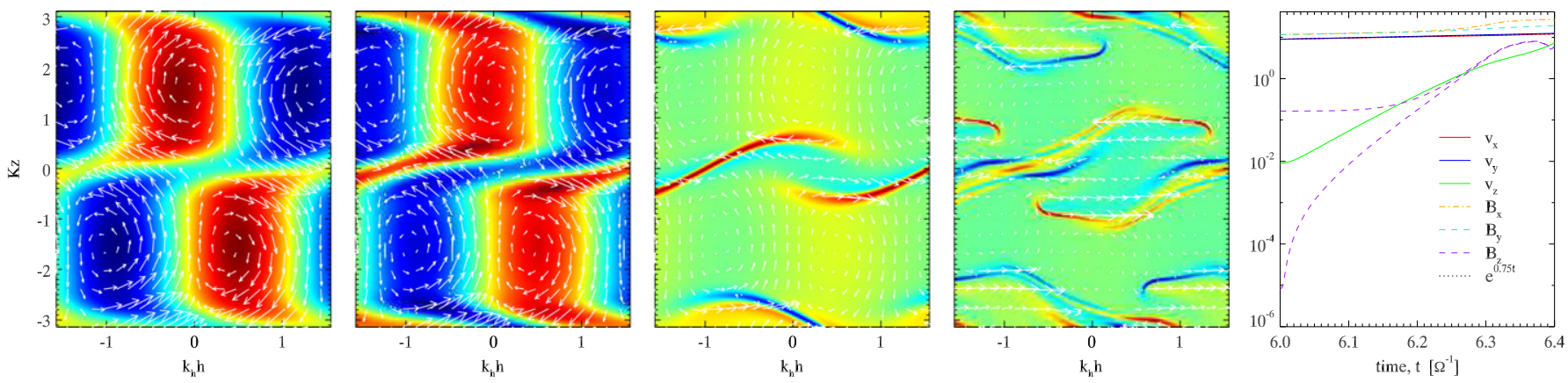

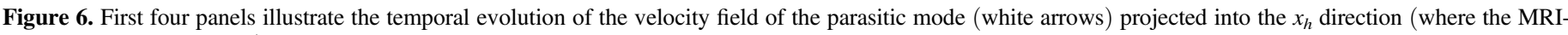

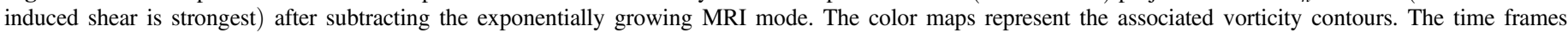
correspond to $t=6.15,6.2,6.3,6.4 \Omega^{-1}$, from left to right. The leftmost panel shows a zoom-in on the growth of the components $v_{x, y, z}$ and $B_{x, y, z}$. 


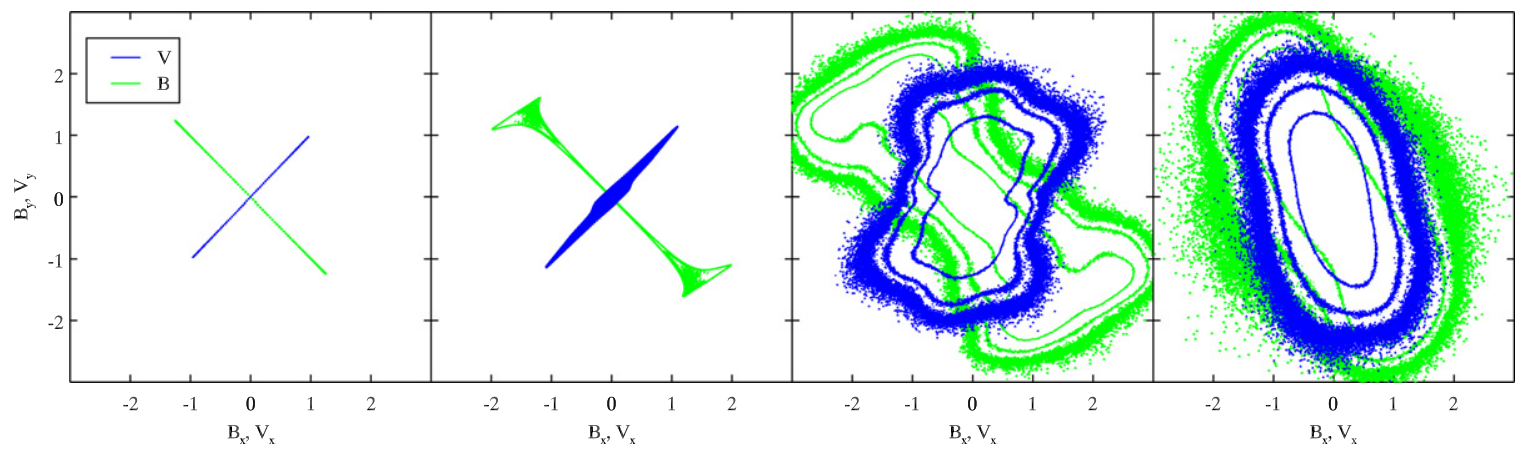

Figure 7. Scatter diagrams of horizontal magnetic and velocity fluctuations $B_{x}, B_{y}$ (green contour) and $v_{x}, v_{y}$ (blue contour) at times $t=6.0,6.5,6.6,15 \Omega^{-1}$, from left to right. At time $t=6.0$, the magnetic and velocity modes are linear, orthogonal, and highly anisotropic. During the growth of the parasite mode $\left(t=6.5 \Omega^{-1}\right)$, the magnetic energy departs from the linear state at high values of magnetic field and begins to partially isotropize. In the saturated state $\left(t=15 \Omega^{-1}\right)$, the flow is still anisotropic. We use the projection introduced in Pessah \& Chan (2008; see, e.g., their Figure 2).

$$
M_{i j}=\frac{\left\langle\delta B_{i} \delta B_{j}\right\rangle}{4 \pi},
$$

where the brackets represent an appropriate spatial average. Each of these real and symmetric tensors can be divided into a deviator and an isotropic part. By normalizing the tensors using their trace, associated with the corresponding energy densities, and then subtracting the isotropic component, we arrive at a single tensor quantity that parametrizes the deviation from isotropy. The Reynolds stress anisotropy tensor (Lumley \& Newman 1977) is defined as

$$
\mathcal{R}_{i j}=\frac{R_{i j}}{\operatorname{Tr}\left(R_{i j}\right)}-\frac{1}{3} \delta_{i j}
$$

where Tr stands for the trace and $\delta_{i j}$ is the Kronecker delta. The Reynolds stress anisotropy tensor provides an important diagnostic and has been used extensively in numerical and experimental studies of hydrodynamic turbulence (Biferale \& Procaccia 2005). Here we extend this idea in order to study MRI-driven turbulence and define the Maxwell stress anisotropy tensor as

$$
\mathcal{M}_{i j}=\frac{M_{i j}}{\operatorname{Tr}\left(M_{i j}\right)}-\frac{1}{3} \delta_{i j} .
$$

The evolution of the global distribution of anisotropy when parasitic modes grow by feeding off the MRI can be quantified via $\mathcal{R}_{i j}$ in Equation (10) by averaging over cubic volumes composed of eight cells. At a given time, this procedure leads to a set of ellipsoidal glyphs, as shown in Figure 9 for three different time snapshots. The orientation and shape of each glyph are controlled by the eigenvectors of $\mathcal{R}$ and their corresponding eigenvalues. The latter, defined in descending order as $\lambda_{1}, \lambda_{2}$, and $\lambda_{3}$, correspond to the principal, medium, and minor axes of the ellipsoidal glyph. The direction of the glyph is aligned along the principal stress eigenvector, i.e., the eigenvector with the largest associated eigenvalue. The leftmost panel shows that all the tensors are aligned in the direction along which the MRI-induced shear is strongest, i.e., $x=y$, indicating that in the late linear stage of the MRI we have highly organized and highly anisotropic flow. The middle panel shows $\mathcal{R}$, after the secondary perturbation has been excited and the parasites have started to grow. The initially highly anisotropic flow has now been partially isotropized, and the principal stress eigenvectors of $\mathcal{R}$ are no longer parallel
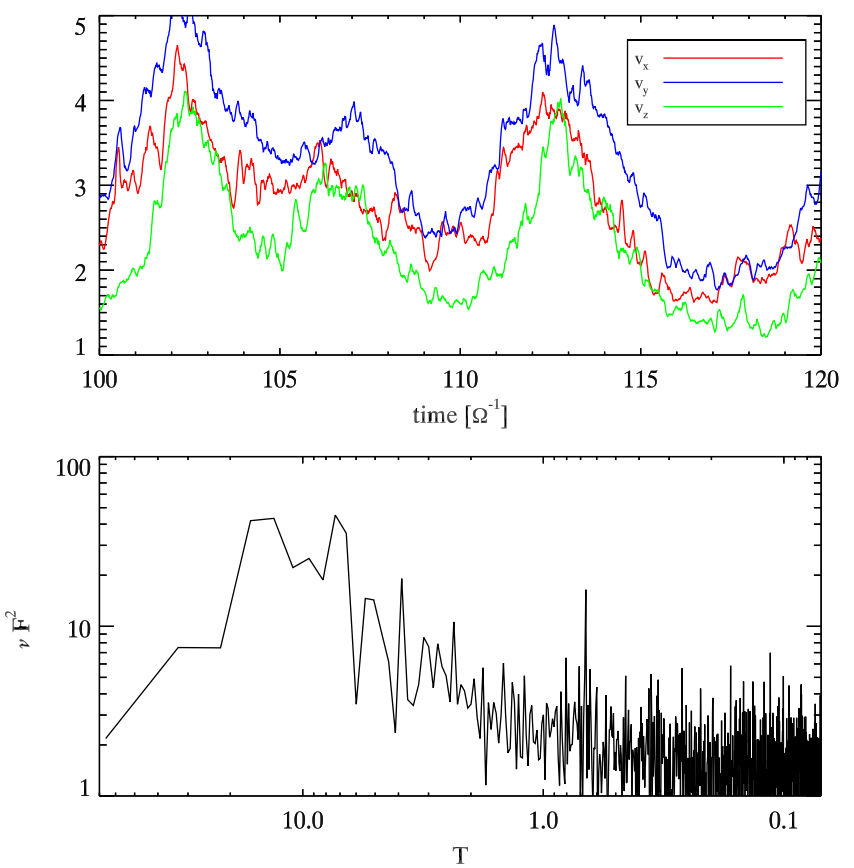

Figure 8. Upper panel: time evolution of the radial and vertical velocity components, $v_{x}$ and $v_{z}$, in the range $t=100-120 \Omega^{-1}$. The radial component $v_{x}$ always starts growing before the vertical component $v_{z}$, which then grows at a faster rate, until both variables peak at the same value and drop to a local minimum. Lower panel: temporal power spectrum resulting from averaging the square of the Fourier transforms corresponding to four independent samples of the time series of the radial component of the velocity.

over the domain. The rightmost panel shows the partially isotropized field in saturation. In this last panel, the system has not yet reached fully developed turbulence, but it has reached the closest approach to isotropy.

In order to probe how the anisotropy on larger scales varies as a function of time in a quantitive way, it is useful to employ invariant quantities. For a traceless tensor, $\lambda_{1}+\lambda_{2}+\lambda_{3}=0$, the degree of anisotropy can be characterized by calculating the two nonzero invariants

$$
\begin{gathered}
\chi_{2}^{\mathcal{R}}=-\left(\lambda_{1}^{2}+\lambda_{2}^{2}+\lambda_{3}^{2}\right), \\
\chi_{3}^{\mathcal{R}}=\lambda_{1} \lambda_{2} \lambda_{3} .
\end{gathered}
$$

By plotting all the possible values of these two independent invariants of the Reynolds stress in the plane spanned by 

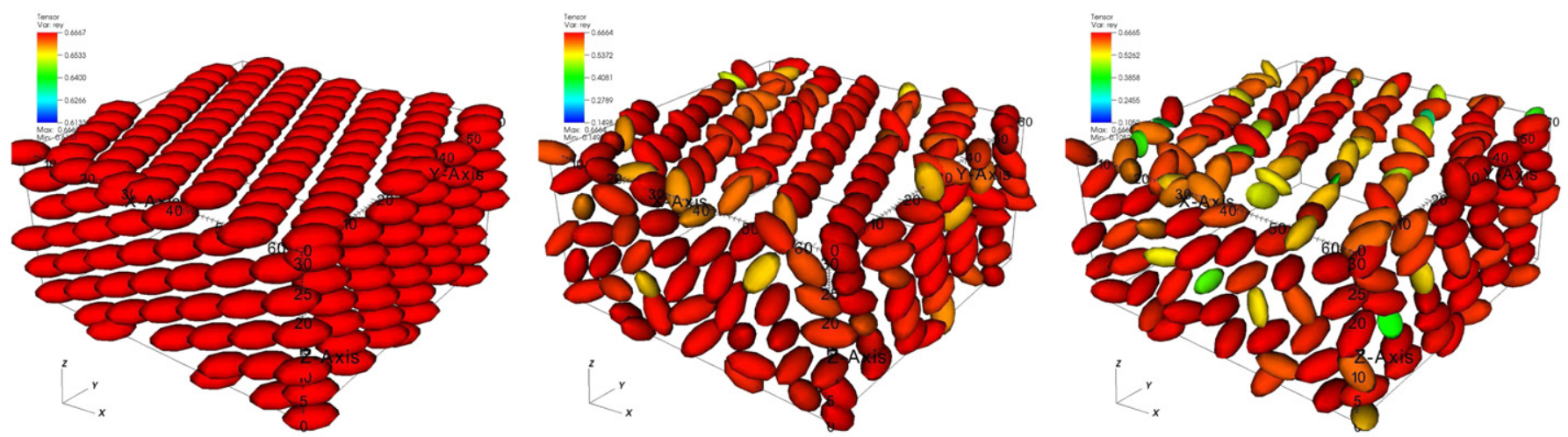

Figure 9. Rendering of the temporal evolution of the Reynolds stress anisotropy tensor, $\mathcal{R}$, at $t=6.2 \Omega^{-1}, t=6.3 \Omega^{-1}$, and $t=6.5 \Omega^{-1}$, from left to right. The lengths principal axes of the ellipsoids are given by the three eigenvalues and are aligned with the direction of the principal eigenvector. The colors indicate the magnitude of the maximum eigenvalue. In the linear phase of the MRI and immediately before saturation, $\mathcal{R}$ is constant and aligned everywhere in the $x=y$ direction, i.e., the direction where the MRI-induced shear is strongest. The second panel shows $\mathcal{R}$ immediately after the onset of the parasitic modes. The parasites have grown and reduced the principal stress and scattered its direction in different regions of the domain. The third panel shows $\mathcal{R}$ well into the saturated phase.
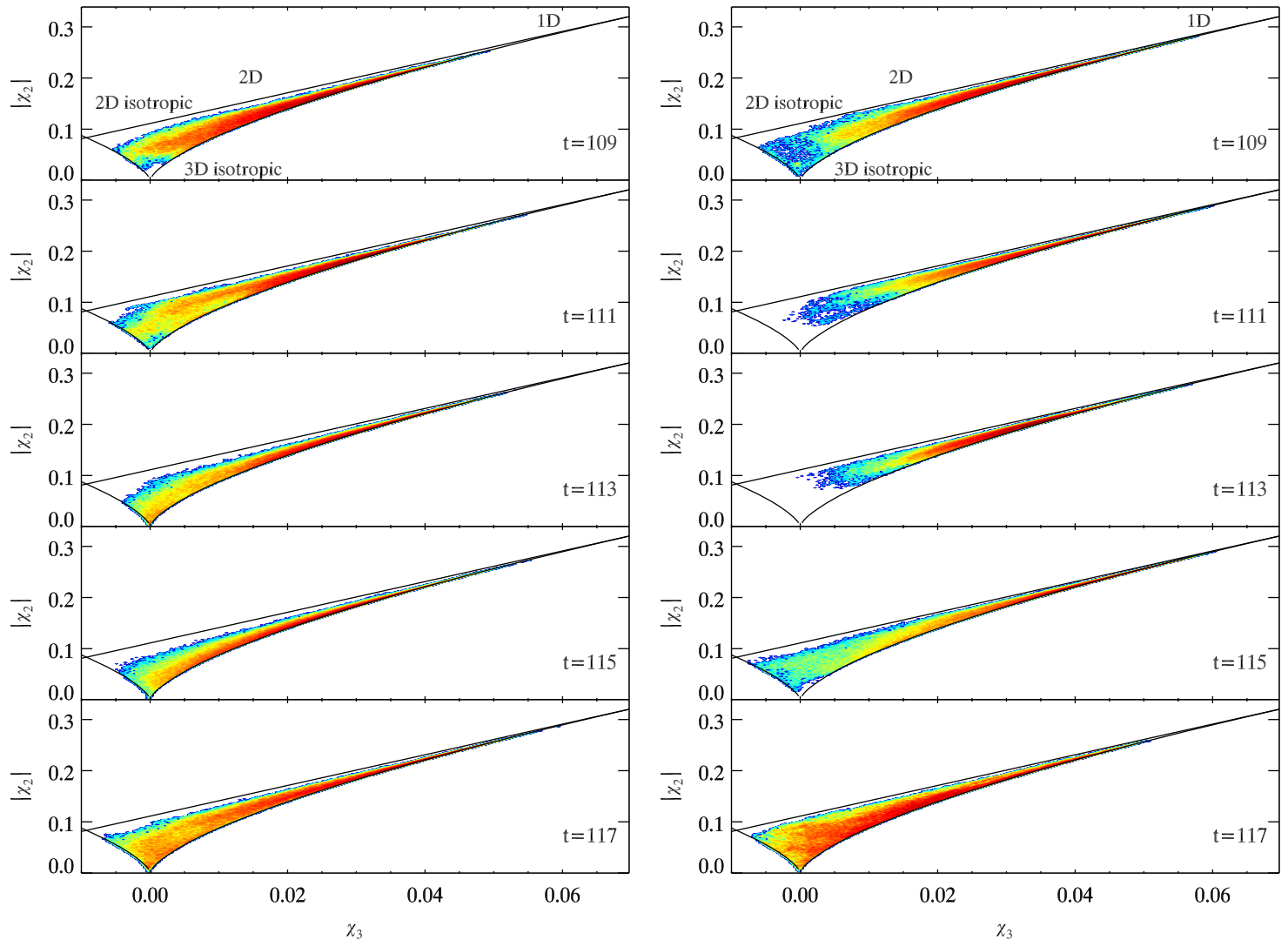

Figure 10. Temporal evolution of the invariant maps for the Reynolds (left column) and Maxwell (right column) stress tensors at $t=40-48 \Omega^{-1}$. The points show the second and third invariants, $\chi_{2}$ and $\chi_{3}$, respectively, of the Reynolds stress anisotropy tensor, for the saturated stage of the MRI. The lines delineate the boundaries on the normalized invariants. The data points near the upper right vertices of the Lumley triangle are indicative of one-component turbulence. The data points close to the lower vertex have three equal eigenvalues, denoting isotropic three-dimensional turbulence, whereas the left vertex denotes two-component turbulence. The color code denotes the number density of subdomains over which the averages were taken that have the same value as the anisotropic invariants.

$\left(\chi_{2}, \chi_{3}\right)$, Lumley \& Newman (1977) found that every realizable Reynolds stress is constrained to lie within three loci that intersect at three vertices. The enclosed deltoid, known as the Lumley triangle, represents all possible values for $\mathcal{R}$. The upper right vertex corresponds to the case of $\lambda_{1}>\lambda_{2}, \lambda_{3}$, and the origin corresponds to isotropy, $\lambda_{1}=\lambda_{2}=\lambda_{3}$. The upper left vertex corresponds to two-component turbulence, with $\lambda_{1}, \lambda_{2}>\lambda_{3}$. The vertical axis in these plots corresponds to the deviation from isotropy, from zero (fully isotropic) through 0.083 (two-component isotropic) to 0.333 (onecomponent). The horizontal axis in the plots correspond to the determinant, which distinguishes between those cases that have the same magnitude of deviation but a different sign.

We provide a quantitative representation of the time evolution of anisotropy on larger scales, by showing in Figure 10 how the Lumley triangles associated with the Reynolds and Maxwell stresses are populated as a function of time. The plots are constructed by taking contours of a $2 \mathrm{D}$ 
histogram of the distribution of vertically averaged tensors. The left column shows the Reynolds stress tensor invariant map at five successive times. As time progresses, the distribution of anisotropy fluctuates, initially increasing between $109<t \Omega^{-1}<113$ and later decreasing between $113<t \Omega^{-1}<117$. The left column of Figure 10 shows the normalized Reynolds stress tensor invariant map, with the second invariant in the vertical axis and the third invariant (determinant) in the horizontal axis. The color map shows the density of points that have associated the pair of values $\left(\chi_{2}, \chi_{3}\right)$ when each stress tensor is averaged in the vertical direction. At $t=119 \Omega^{-1}$, the stress tensors have a peak that is far from the origin. The highest density (shown in red) is offset from the origin, which is consistent with anisotropic turbulence. The majority of points are distributed close to the 1D-3D locus, with almost no points on the $1 \mathrm{D}-2 \mathrm{D}$ locus. As time progress, at $t=111 \Omega^{-1}$ and $t=113 \Omega^{-1}$ the distribution of anisotropy shifts along the 1D-3D locus, further from isotropy (i.e., the origin). At $t=113 \Omega^{-1}$, the peak of anisotropy is reached and almost no power is present near the origin. At $t=115 \Omega^{-1}$, the distribution of invariants shifts back toward the origin, indicating a return toward isotropy. The peak of the distribution remains far from the origin, however. At $t=117 \Omega^{-1}$, the distribution shifts even further toward the origin.

This effect is even more pronounced in the Maxwell stress tensor (right column of Figure 10), which shows a strong increase in anisotropy between $109<t \Omega^{-1}<113$, which then reverses between $113<t \Omega^{-1}<117$. The examination of other time frames (not reproduced here) reveals that this pattern of increase and decrease in anisotropy is quasi-periodic, in agreement with Figure 8 .

\subsection{Time-dependent, Anisotropic Power Spectral Distributions}

A number of works have studied the Fourier spectrum of MRI-driven turbulence (for early studies see, e.g., Hawley et al. 1995; for more recent work see Fromang 2010; Lesur \& Longaretti 2011; Nauman \& Blackman 2014). In order to visualize the distribution of power as a function of scale, it is customary to plot either spherical shell-averaged spectra or 2D slices in the planes perpendicular to the directions given by $\hat{\boldsymbol{k}}_{x}, \hat{\boldsymbol{k}}_{y}$, and $\hat{\boldsymbol{k}}_{z}$. The second approach reveals that the turbulence is highly anisotropic, rendering the first approach suspect. In either case, temporal averages over many orbital timescales are usually invoked in order to obtain smooth spectra. However, as we have shown in the previous section, the degree of anisotropy of the turbulence varies with time significantly.

In order to gain a deeper understanding of the temporal evolution of the anisotropy of the turbulence in Fourier space, we pursue a different approach, as follows. We demonstrate the procedure by computing the Fourier spectrum ${ }^{2}$ associated with the magnetic energy density in the early stages after the saturation of the linear instability and in the turbulent state. It is useful to visualize the results by selecting $2 \mathrm{D}$ cuts in the $3 \mathrm{D}$

\footnotetext{
2 Standard Fourier analysis requires a periodic domain, and the shearing box domain that we employ is only strictly periodic in the azimuthal and vertical directions. The radial direction, which is only shearing periodic, becomes strictly periodic only at specific times (Hawley et al. 1995). In order to compute Fourier transforms at arbitrary times, it is necessary to first transform in the $x$-direction and then shift the appropriate phase before taking the transform in the $y$ - and $z$-directions. This procedure is explained in detail in, e.g., Heinemann \& Papaloizou (2009).
}

Fourier space. The anisotropic nature of the turbulence in the $\left(k_{x}, k_{y}\right)$ plane presents a natural direction to define two perpendicular directions in this plane. We define $\hat{\boldsymbol{k}}_{1}$ as the unit wavevector corresponding to the direction of maximum anisotropy in the plane spanned by $\left(k_{x}, k_{y}\right)$. The unit vectors $\hat{\boldsymbol{k}}_{3}=\hat{\boldsymbol{k}}_{z}$ and $\hat{\boldsymbol{k}}_{3} \times \hat{\boldsymbol{k}}_{1}$ complete the orthonormal triad. Note that the directions provided by $\hat{\boldsymbol{k}}_{1}$ and $\hat{\boldsymbol{k}}_{2}$ depend in general on time!

Figure 11 shows the $2 \mathrm{D}$ cuts along the three different planes perpendicular to $\hat{\boldsymbol{k}}_{1}, \hat{\boldsymbol{k}}_{2}$, and $\hat{\boldsymbol{k}}_{z}$ for two different times, corresponding to the early times after the saturation of the linear phase of the MRI $\left(t=10 \Omega^{-1}\right.$, upper row $)$ and the fully developed turbulent state $\left(t=260 \Omega^{-1}\right.$, lower row $)$. In order to quantify the degree of anisotropy, at a given time, we performed a 2D Gaussian fit, which is overplotted in each panel. The Gaussian fit is carried out by identifying the peak of the power spectral density and then fitting a 1D Gaussian along the direction of maximum anisotropy and another 1D Gaussian in the direction perpendicular to it. The width of these two Gaussians determines the semimajor axis of the $2 \mathrm{D}$ ellipses that are plotted over the data.

At time $t=10 \Omega^{-1}$, the parasite mode has reached the peak of its super-exponential growth, as attested by the presence of significant power in non-axisymmetric modes. The distribution of power in the $\left(k_{2}, k_{z}\right)$ plane is mostly isotropic, the 2D Gaussian fit has an aspect ratio of 1.12. On the other hand, the Gaussian fits performed in the $\left(k_{1}, k_{z}\right)$ and $\left(k_{x}, k_{y}\right)$ planes show aspect ratios of $\sim 1.88,3.12$ respectively. In the fully developed turbulent state, at $t=260 \Omega^{-1}$, the flow is highly anisotropic in the $\left(k_{1}, k_{z}\right)$ plane. The aspect ratios of the Gaussian fits in the $\left(k_{1}, k_{z}\right),\left(k_{2}, k_{z}\right)$, and $\left(k_{x}, k_{y}\right)$ planes are, respectively, 2.63, 1.16, and 3.59. The anisotropy of the spectrum has therefore increased significantly from the late linear evolution of the MRI to the ensuing turbulent state.

The contrast between the energy spectral density obtained using spherical shell-averaged values versus $1 \mathrm{D}$ profiles in each of the three planes described above is depicted in the rightmost panel in Figure 11. At early times, i.e., soon after the parasitic modes have disrupted the highly organized flow driven by the exponential growth of the MRI, the 1D spectra along the directions $\hat{\boldsymbol{k}}_{1}$ and $\hat{\boldsymbol{k}}_{2}$ is not exceedingly different, although it is clear that there is more power along $\hat{\boldsymbol{k}}_{1}$. However, at later times, in the fully developed turbulent regime, the turbulent power along $\hat{\boldsymbol{k}}_{1}$ is almost two orders of magnitude higher than the power in the direction perpendicular to it throughout most of the scales. In this case, the shell average is unable to provide a good representation of the anisotropic power spectrum of the turbulence. In both cases, averaging over spherical shells masks the spread of almost two orders of magnitude in power along the directions given by $\hat{\boldsymbol{k}}_{1}$ and $\hat{\boldsymbol{k}}_{2}$.

The distribution of power characterizing the Maxwell stress in Fourier space can be analyzed in a similar way that we have analyzed the magnetic energy density. The results are shown in Figure 12. As observed for the magnetic energy density, the Maxwell stress is also highly anisotropic and the degree of anisotropy varies significantly with time. Note that the fast decrease in power along the $k_{x}$ and $k_{y}$ directions, i.e., the usual directions along which 1D PSD cuts are plotted, is even steeper for the Maxwell stress than for the magnetic energy. This implies that if we are interested in characterizing the degree of anisotropy of the stress is even more critical to analyze the 

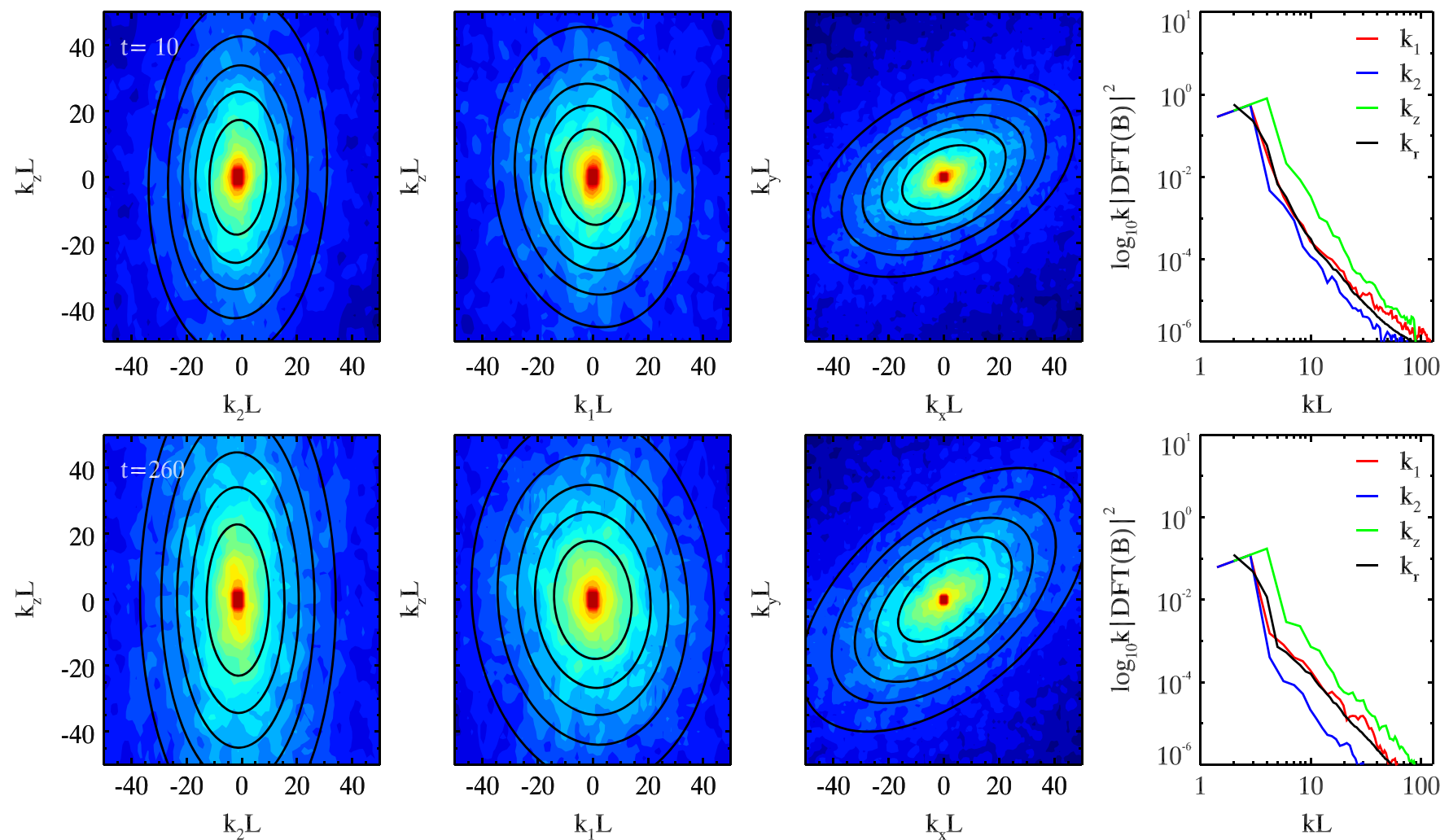

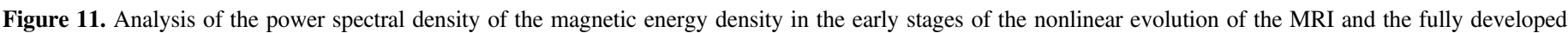

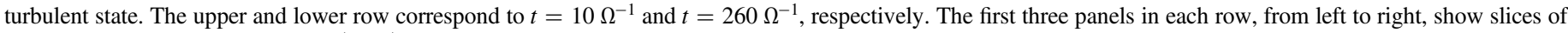

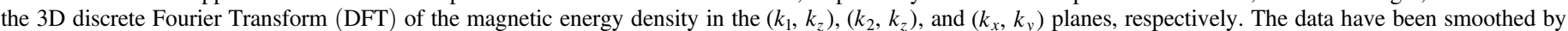

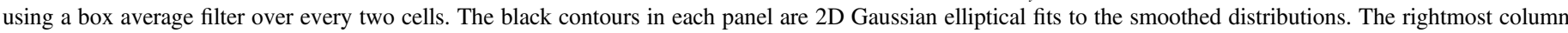

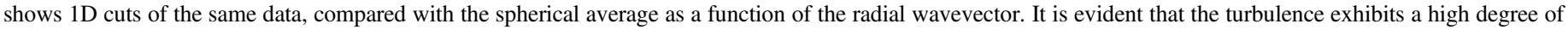
anisotropy. In particular, there is difference of almost two orders of magnitude in power between the 1D spectra along $k z$ and $k_{2}$.

spectra along $\hat{\boldsymbol{k}}_{1}$ and $\hat{\boldsymbol{k}}_{2}$, which provide a better characterization of the 3D distribution of power in Fourier space.

\subsection{Defining Robust Averages In Fourier Space}

It is evident that the distribution of power in Fourier space is highly anisotropic and time dependent. Therefore, in order to provide a robust characterization of the turbulent spectrum we proceed as follows. At a fixed time, we obtain the 3D PSD in Fourier space and define the direction of maximum anisotropy in horizontal planes. This is accomplished by fitting a 2D Gaussian ellipse to the distribution in the $\left(k_{x}, k_{y}\right)$ plane and defining the direction of maximum anisotropy as $\boldsymbol{k}_{1}$ and the two orthogonal directions $\boldsymbol{k}_{2}$ and $\boldsymbol{k}_{3}$. The 1D power spectra along each of these directions are readily obtained. This procedure is repeated for different times, and the resulting 1D spectra can be properly averaged along each independent direction.

The average power spectra of the magnetic energy density obtained in this way are shown in Figure 13. Most of the power is concentrated in large scales, and a power-law behavior is observed beyond a characteristic wavenumber, which is different along each direction. In order to quantify the steepness of these profiles, we fitted power-law $k^{-\alpha_{i}}$ distributions to $k|\operatorname{DFT}(B)|^{2}$ along each independent direction $i=1,2,3$. We obtained $\alpha_{1} \sim 2.8, \alpha_{2} \sim 2.9, \alpha_{z} \sim 3.2$. For comparison, we also plotted the power spectra that result from a standard spherical shell average. This corresponds to $\alpha_{r}=3$. The orientation of the ellipsoid in Fourier space can be characterized by the angle subtended between $\boldsymbol{k}_{1}$ and $\boldsymbol{k}_{x}$. The average value of this angle is about $35^{\circ}$. The distribution of the values of this angle is shown in the inset.

The fact that the distribution of power in MRI-driven turbulence is anisotropic in Fourier space was already indicated by Hawley et al. (1995). Their Figure 4 shows that most of the power in the magnetic field resides in modes along $k_{z}$, in qualitative agreement with our results. Hawley et al. (1995) also note a steepening at higher values of $k$ due to numerical diffusion, which we do not find in our simulations, possibly owing to the incompressible pseudo-spectral method being less diffusive than the second-order scheme (see, e.g., Figure 1 of Brandenburg 2001). Our results can also be compared with those of Lesur \& Longaretti (2011), who defined a spherical average and found a $3 / 2$ slope for both the magnetic and kinetic energy spectra in the $1<k<10$ range, with a subsequent steeper falloff in the range $10<k<100$. In those simulations, there does not seem to be a clear sign of an inertial range. The simulations in Lesur \& Longaretti (2011) used a higher value of $\beta=q \Omega L / B^{2}=10^{3}$ than our $\beta \sim 10$, a slightly different aspect ratio of $4 \times 4 \times 1$, and a broadband noise excitation. The fact that we have chosen the value of $\beta$ such that a single wavelength of the fastest-growing MRI mode fits in the domain may have helped to set an effectively larger injection scale, possibly resulting in the presence of a larger inertial range, as seen in Figure 13.

It is worth pointing out that most of the turbulent power, in both the magnetic energy density and the Maxwell stress, resides in modes with wavevectors along $\boldsymbol{k}_{z}$, i.e., the direction 

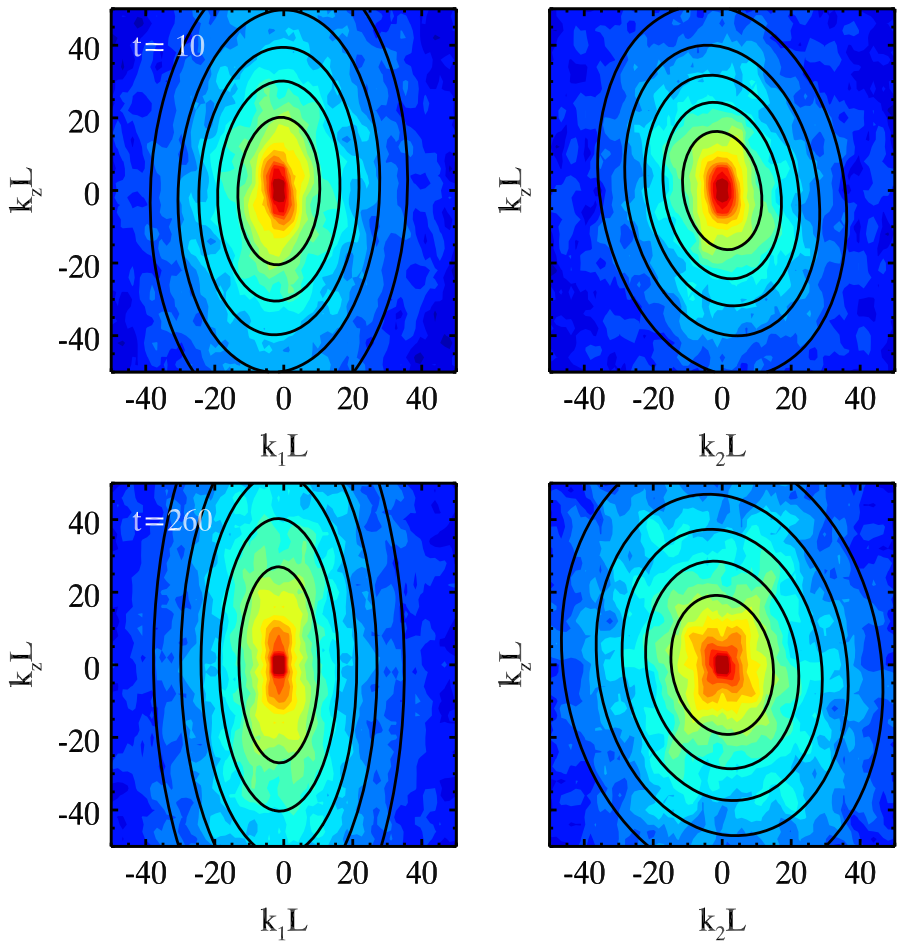
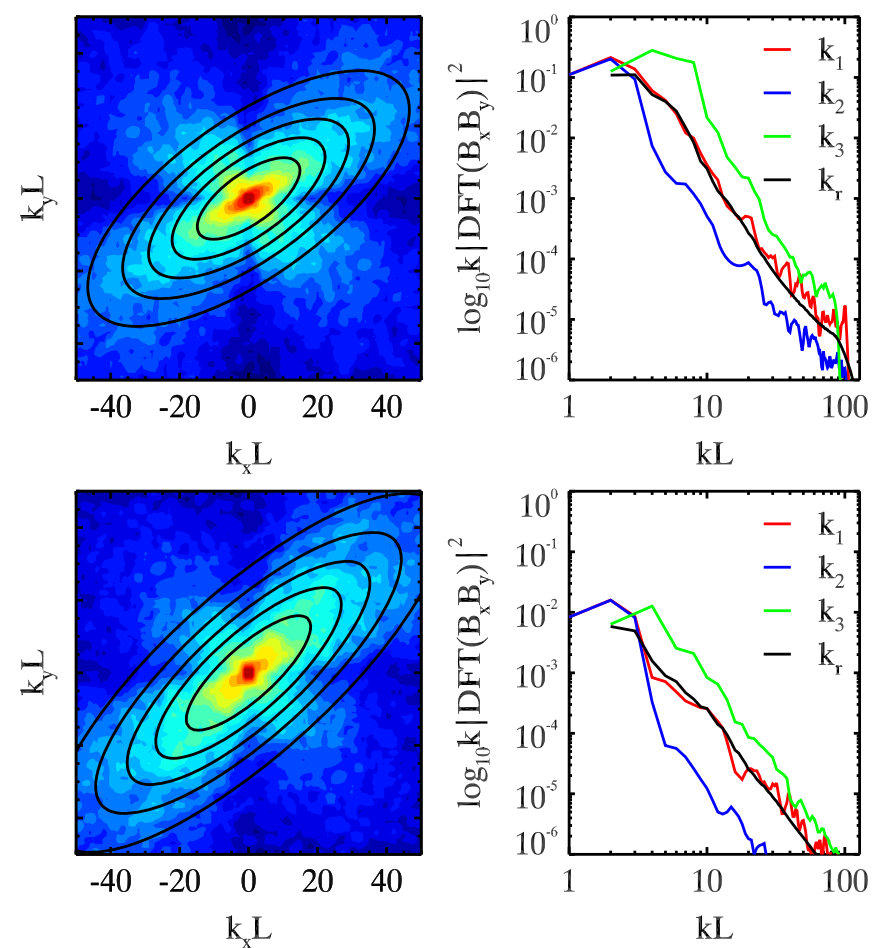

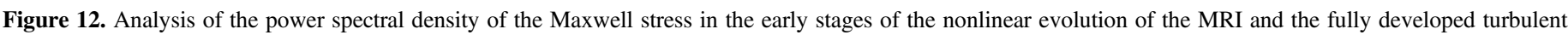

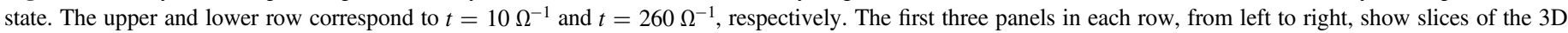

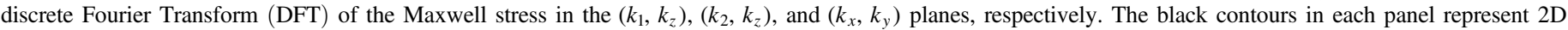

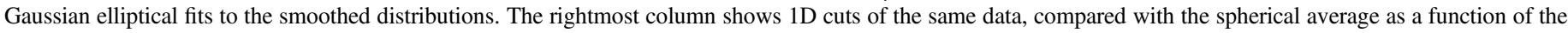
radial wavevector. The power spectra of the Maxwell stress exhibits a high degree of anisotropy, which depends on time.

perpendicular to the shear, with the contribution from nonaxisymmetric modes being subdominant. This could be due to the fact that we have considered a rather strong vertical magnetic field (see, e.g., Pessah et al. 2007). Whether this ordering of power in Fourier space is a generic feature of MRIdriven turbulence in more general settings, e.g., in the presence of weaker fields and/or more general magnetic field geometries, or stratification will be addressed in subsequent work.

\section{SUMMARY AND DISCUSSION}

In this paper we have shed light on the nature of MRI-driven turbulence by studying in detail the temporal evolution of various indicators that quantify the degree of anisotropy of the magnetized flow. We accomplished this task by starting with a well-defined numerical experiment and analyzing the outcome in ways that have not been previously explored in the context of MRI-driven turbulence.

We analyzed the saturation of the MRI via parasitic instabilities and the subsequent transition to anisotropic turbulence by means of 3D nonlinear MHD simulations carried out with the pseudo-spectral code SNOOPY (Lesur \& Longaretti 2009). We found that the exponential growth of the MRI is halted by the superexponential growth of a parasite instability, which corresponds to an exponentially driven $\mathrm{KH}$ mode (see Section 3). These findings are in general agreement with semi-analytical studies by Goodman \& Xu (1994) and Pessah (2010), as well as the numerical studies by Latter et al. (2009), Longaretti \& Lesur (2010), and Obergaulinger et al. (2009). Once the nonaxisymmetric secondary instability reaches an amplitude similar to the MRI mode, the highly organized, anisotropic flow driven by the MRI becomes more isotropic and breaks down into fully developed MHD turbulence (see Section 4).

We showed that MRI-driven turbulence is characterized by quasi-periodic fluctuations in the radial and vertical velocity components throughout the duration of the simulations (Figure 8). In particular, $v_{x}$ is tracked by $v_{z}$, with $v_{z}$ increasing until it reaches an amplitude equal to $v_{x}$, whereupon they both decline together. This behavior mimics the growth of $v_{x}$ followed by $v_{z}$ observed during the phase where parasitic instabilities halt the growth of the MRI (Figure 4). This suggests that a turbulent version of the process that takes place in the late stages of the linear regime of the instability could be at work. This cyclic behavior is indicative of a strong temporal variation in the anisotropic properties of the flow. We argued that more refined statistical tools are needed in order to shed light on the anisotropic nature of MRI-driven turbulence.

In order to quantify and classify the anisotropy characterizing MRI-driven turbulence (see Section 5.1), we adopted a technique employed in hydrodynamic turbulence, which is built on invariant analysis (Lumley \& Newman 1977). In particular, we extended the use of these tools to analyze the properties of the Maxwell stress, which is the dominant source of angular momentum transport in ideal MHD. The invariant analysis shows a strong anisotropy, with most of the power concentrated in the one-component regime, with brief sporadic transitions toward two-component and three-component turbulence. We noted that the fact that the level of anisotropy fluctuates on timescales comparable to $\sim 10 \Omega^{-1}$ suggests that averaging Fourier spectra over long time periods in order to obtain smooth spectra may influence the derived spectrum. 

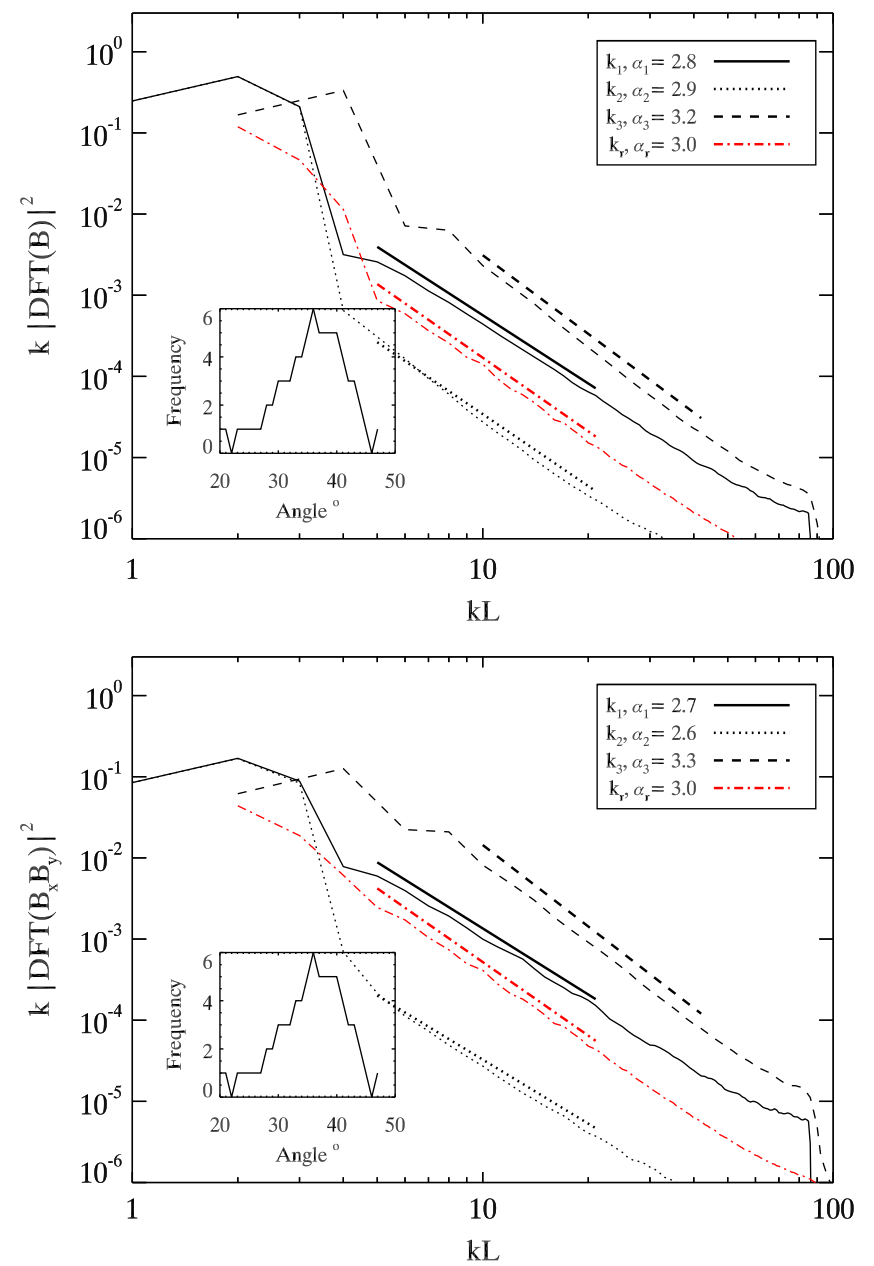

Figure 13. Upper panel: time-averaged plot of the distribution of energy in the directions given by $\hat{\boldsymbol{k}}_{1}, \hat{\boldsymbol{k}}_{2}$ and $\hat{\boldsymbol{k}}_{3}=\hat{\boldsymbol{k}}_{z}$ averaged over $260 \Omega^{-1}$. The corresponding shell-averaged spectrum is also shown for comparison. At high wavenumbers the power-law behavior exhibited by $k|\mathrm{DFT}(B)|^{2}$ can be described by $k^{-\alpha_{i}}$ with $\alpha_{1} \sim 2.8, \alpha_{2} \sim 2.9, \alpha_{z} \sim 3.2$. The index obtained for the shell-averaged spectrum is $\alpha_{r}=3$. The inset shows the distribution of values of the angle subtended by the direction of maximum anisotropy in the $\left(k_{x}, k_{y}\right)$ plane. Lower panel: time-averaged plot of the distribution of Maxwell stress. At high wavenumbers the power-law behavior exhibited by $k\left|\operatorname{DFT}\left(B_{x} B_{y}\right)\right|^{2}$ can be described by $k^{-\alpha_{i}}$ with $\alpha_{1} \sim 2.7, \alpha_{2} \sim 2.6, \alpha_{z} \sim 3.3$.

We also investigated the impact associated with using shell averages to characterize the inherently anisotropic MRI-driven turbulence (see Section 5.3). This technique, which carries with it the implicit assumption of isotropy in Fourier space, has been widely adopted to characterize MRI-driven turbulence (Hawley et al. 1995; Workman \& Armitage 2008; Fromang 2010). Our analysis consisted of dissecting the 3D Fourier spectrum, using 2D cuts along planes selected according to the direction of maximum anisotropy. We found that the spectral state of turbulence is far from spherically symmetric and that, in agreement with the invariant analysis, the degree of anisotropy in Fourier space depends on time as well.

This led us to propose what we believe to be a more robust way to average Fourier spectra, by consistently stacking 1D spectra along the directions of maximum anisotropy at any given time. The result of this process is shown in Figure 13, which also shows that the direction of maximum anisotropy in the plane $\left(k_{x}, k_{y}\right)$ oscillates around $35^{\circ}$, with respect to $k_{x}$. The distribution of the angle subtended by the direction of maximum anisotropy, shown in the inset, reflects the timedependent nature of the anisotropy in Fourier space.

Using this procedure, we provided statistical significance to a fact that was already evident in the spectra taken at two different times (see, e.g., Figure 11). In the turbulent state, the power along the three independent directions that show maximum anisotropy differs by several orders of magnitude over most scales, except the largest ones. This indicates that the spherically averaging technique to seek scaling relationships for MRI-driven turbulence may distort the underlying, inherently anisotropic turbulent spectrum. In particular, the identification of an inertial range for differentially rotating MHD turbulence may be hampered by the use of spherical averages to seek isotropic scaling laws, which are at odds with the strong anisotropy that leads naturally to efficient angular momentum transport.

This work focused on a simple configuration, with a vertical magnetic field for which the wavelength of the fastest-growing mode matches the vertical extent of the unstratified domain. Cyclic behavior in numerical simulations of MRI-driven turbulence has been reported by a number of previous works involving different settings. Considering a simulation domain threaded by a toroidal magnetic field, Herault et al. (2011) sought exact nonlinear dynamo cycles numerically. These solutions are characterized by counterrotating cellular structures that naturally lead to periodic variations in the flow properties. In the context of stratified shearing box simulations, cyclic behavior is usually attributed to the propagation of dynamo waves (Davis et al. 2010; Gressel 2010; Simon \& Armitage 2012). Understanding the relationship between the results presented in this paper and previous works will entail employing the tools that we have developed in simulations with different physical settings considering, for example, more general magnetic field geometries and stratification.

Characterizing the anisotropic nature of MRI-driven turbulence is a step forward toward developing a theory for turbulent transport in magnetized flows in astrophysical disks. Recognizing the need to move beyond the assumption of an isotropic turbulent cascade demands the development of more sophisticated theoretical tools in order to accomplish this goal. In this context, we note that Mamatsashvili et al. (2014) have developed a set of tools for studying shear MHD turbulence that would be interesting to build upon in order to gain further insight into the dynamics of MRI-driven turbulence.

The authors thank G. Lesur, C. McNally, O. Gressel, J. Oishi, G. Mamatsashvili, and A. Nordlund for helpful discussions. The results presented in this paper were partly achieved using the PRACE Research Infrastructure resource JUQUEEN based in Germany at the Jülich Supercomputing Centre. The authors also acknowledge DCSC/KU and ICHEC for computational facilities and support. The research leading to these results has received funding from the European Research Council under the European Union's Seventh Framework Programme (FP/2007-2013) under ERC grant agreement 306614 (M.E.P.). The authors also acknowledge support from the Young Investigator Programme of the Villum Foundation. 


\section{REFERENCES}

Balbus, S. A., \& Hawley, J. F. 1991, ApJ, 376, 214

Bartlett, M. S. 1948, Natur, 161, 686

Biferale, L., \& Procaccia, I. 2005, PhR, 414, 43

Bodo, G., Mignone, A., Cattaneo, F., Rossi, P., \& Ferrari, A. 2008, A\&A, 487, 1

Brandenburg, A. 2001, arXiv:0109497v1

Brandenburg, A., Nordlund, Å., Stein, R. F., \& Torkelsson, U. 1996, ApJ, 458, L45

Davis, S., Stone, J. M., \& Pessah, M. E. 2010, ApJ, 713, 52

Ebrahimi, F., Prager, S. C., \& Schnack, D. D. 2009, ApJS, 698, 233

Frank, A., Ryu, D., Jones, T. W., \& Gaalaas, J. B. 1996, ApJS, 460, 777

Frank, J., King, A., \& Raine, D. J. 2002, in Accretion Power in Astrophysics (3rd ed.; Cambridge: Cambridge University Press)

Fromang, S. 2010, A\&A, 514, L5

Goodman, J., \& Xu, G. 1994, ApJ, 432, 213

Gressel, O. 2010, MNRAS, 405, 41

Hawley, J. F., Gammie, C. F., \& Balbus, S. A. 1995, ApJ, 440, 742

Heinemann, T., \& Papaloizou, J. 2009, MNRAS, 397, 52

Herault, J., Rincon, F., Cossu, C., et al. 2011, PhRvE, 84, 036321

Jamroz, B., Julien, K., \& Knobloch, E. 2008a, PhyS, T132, 014027

Jamroz, B., Julien, K., \& Knobloch, E. 2008b, AN, 329, 675

Jones, T. W., Gaalaas, J. B., Ryu, D., \& Frank, A. 1997, ApJ, 482, 230

Julien, K., \& Knobloch, E. 2006, EAS Publications Series, 21, 81

Julien, K., \& Knobloch, E. 2010, RSPTA, 368, 1607

Knobloch, E., \& Julien, K. 2005, PhFl, 17, 4106
Latter, H. N., Lesaffre, P., \& Balbus, S. A. 2009, MNRAS, 394, 715

Lesur, G., \& Longaretti, P.-Y. 2009, A\&A, 504, 309

Lesur, G., \& Longaretti, P.-Y. 2011, A\&A, 528, A17

Liverts, E., Shtemler, Y., Mond, M., Umurhan, O. M., \& Bisikalo, D. V. 2012, PhRvL, 109, 224501

Longaretti, P.-Y., \& Lesur, G. 2010, A\&A, 516, A51

Lumley, J. L., \& Newman, G. R. 1977, JFM, 82, 161

Lynden-Bell, D., \& Pringle, J. E. 1974, MNRAS, 168, 603

Lyra, W., \& Klahr, H. 2011, A\&A, 527, A138

Malagoli, A., Bodo, G., \& Rosner, R. 1996, ApJ, 456, 708

Mamatsashvili, G. R., Gogichaishvili, D. Z., Chagelishvili, G. D., \& Horton, W. 2014, PhRvE, 89, 043101

Mignone, A., Zanni, C., Tzeferacos, P., et al. 2012, ApJS, 198, 7

Nauman, F., \& Blackman, E. G. 2014, MNRAS, 441, 1855

Obergaulinger, M., Cerdá-Durán, P., Müller, E., \& Aloy, M. A. 2009, A\&A, 498, 241

Pessah, M. E. 2010, ApJ, 716, 1012

Pessah, M. E., \& Chan, C.-K. 2008, ApJ, 684, 498

Pessah, M. E., Chan, C.-K., \& Psaltis, D. 2006, MNRAS, 372, 183

Pessah, M. E., Chan, C.-K., \& Psaltis, D. 2007, ApJL, 668, L51

Pessah, M. E., \& Goodman, J. 2009, ApJL, 698, L72

Shakura, N. I., \& Sunyaev, R. A. 1973, A\&A, 24, 337

Simon, J. B., \& Armitage, P. J. 2012, MNRAS, 422, 2685

Tatsuno, T., \& Dorland, W. 2008, AN, 329, 688

Umurhan, O., Regev, O., \& Menou, K. 2007, PhRvL, 98, 034501

Vishniac, E. T. 2009, ApJ, 696, 1021

Workman, J. C., \& Armitage, P. J. 2008, ApJ, 685, 406 